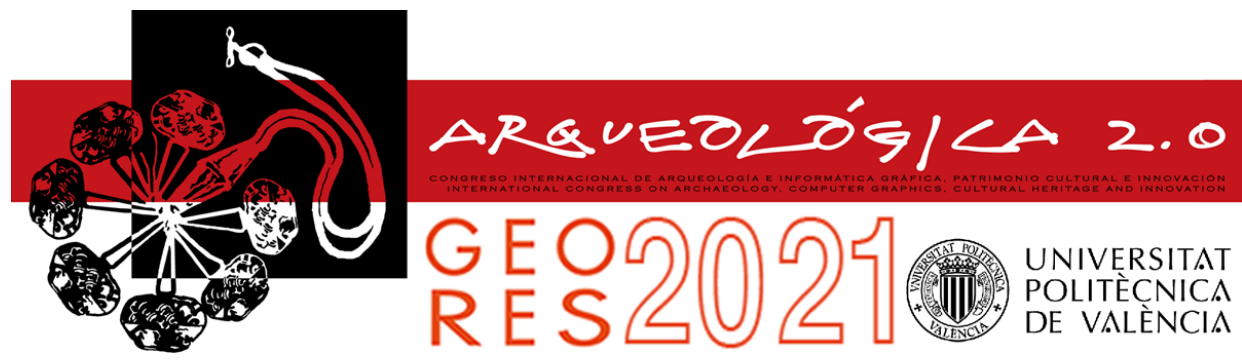

Proceedings of the joint international event $9^{\text {th }}$ ARQUEOLÓGICA

$2.0 \& 3^{\text {rd }}$ GEORES

Valencia (Spain).

26-28 April 2021

\title{
COPERNICUS EARTH OBSERVATION FOR CULTURAL HERITAGE
}

\author{
Athos Agapiou ${ }^{a, b,{ }^{*},}$ Vasiliki Lysandrou ${ }^{a, b}$, Branka Cucac \\ a Department of Civil Engineering and Geomatics, Faculty of Engineering and Technology, Cyprus University of Technology, Saripolou \\ 2-8, 3036 Limassol, Cyprus. athos.agapiou@cut.ac.cy; vasiliki.lysandrou@cut.ac.cy \\ ${ }^{b}$ Eratosthenes Centre of Excellence, Saripolou 2-8, 3036 Limassol, Cyprus.
}

${ }^{\mathrm{c}}$ Department of Architecture, Built Environment and Construction Engineering, Politecnico di Milano, Piazza Leonardo da Vinci 32, 20133 Milan, Italy. branka.cuca@polimi.it

\begin{abstract}
:
This article summarises the European Union's Earth Observation Copernicus Programme's contribution and other satellitebased platforms for cultural heritage applications. It highlights the results of some national and European projects conducted in this domain. Even though the Copernicus Programme has recently been introduced for cultural heritage use (i.e., after the second half of this decade), the full, free, and open data policy, known as FFO, has increased the number of relevant applications. Since then, several studies dedicated to cultural heritage monitoring, management, etc., have been presented in the literature. Despite the medium spatial resolution of the radar and optical Sentinel sensors, their hightemporal revisit time combined with other legacy space programmes (e.g., the Landsat space programme) can provide useful information to local and regional stakeholders, and other national agencies. New developments in the domain of image processing like the use of big-data earth observation cloud platforms already showcased the potentials for dedicated space-based cultural heritage services. However, this technological shift remains still within the scientific and theoretical research domain. Therefore, much effort is still needed for the implementation of national policies.
\end{abstract}

Keywords: Copernicus Programme, cultural heritage, Sentinel sensors, remote sensing, satellite observation, heritage management

\section{The Copernicus Programme}

European Union's Earth Observation Copernicus Programme is coordinated and managed by the European Commission (EC) with the support of the European Space Agency (ESA), the European Union's Member States and European Union's Agencies (Copernicus, 2020). The scope of this multi-billion spacebased programme is to provide various digital geospatial information services and datasets, systematically acquired from satellite sensors. With almost global coverage, these calibrated datasets can be integrated with other relevant ground-based, airborne, and seaborne measurements and remotely-sensed datasets.

Today, the Copernicus Programme is composed of different segments, including the space infrastructure and the ground segment. Besides, satellite-based services are provided through the exploitation of these datasets (Satellite Components, 2020).

The Copernicus Earth observation satellites are divided into two groups of missions. The Copernicus space infrastructure is built upon a family of satellites and instruments called "Sentinels" (Sentinel Overview, 2020). This study focuses on the first two instruments, namely the Sentinel-1 (2020) and the Sentinel-2 (2020) sensors. These sensors can provide radar and optical images systematically. Both sensors were employed in the recent past for different cultural heritage applications and heritage management purposes.

Beyond the Sentinel sensors, the Contributing Missions which are operated by National, European, or International organisations - provide a series of other supportive data, which can further integrate the various Copernicus services. Contributing Missions data are important for quite a few satellite-based applications as these sensors usually provide higher spatial resolution images, a key parameter for particular case-studies.

The ground Copernicus segment is delivered by international agencies (ESA and EUMETSAT) and national public and private facilities. It provides the "mission control", that turns the Sentinel satellites and facilities data into Copernicus Services products.

Currently, six different thematic Copernicus-based services can be found. These are the marine, atmosphere, land, climate change, emergency, and security (Copernicus Services, 2020). Copernicus services are operational since 2014 and support, among others, policies such as environment, transport, energy, 
agriculture and forestry, migration, border security, maritime safety, disaster management, urban planning, development, energy, and fight against climate change.

Access to the Copernicus datasets and services is of fundamental importance for any exploitation concerning cultural heritage. A particular aspect of the Copernicus Programme relies on its FFO policy (full, free, and open policy) (European Comission, 2018). Therefore, Copernicus data and products are freely and openly accessible to end-users, with the possibility to use and reuse these data and information for addressing specific needs.

At the same time, the FFO policy ensures the most comprehensive re-use of these datasets. Open data drives growth by stimulating the creation of firms that reuse such data in innovative ways.

The current paper presents technological shifts that have observed in the last years in the domain of earth observation for documentation, mapping and monitoring of cultural heritage environments, including big-data cloud platforms. The paper presents an overview of the contribution of the Copernicus Programme to cultural heritage, through the results of two on-going research projects, namely the "Copernicus Earth Observation Big Data for Cultural Heritage" (in short NAVIGATOR http://web.cut.ac.cy/navigator/), and the "Portal for heritage buildings integration into the contemporary built environment" (in short PERISCOPE https://uperiscope.cyi.ac.cy/). Both projects are cofinanced by the European Regional Development Fund and the Republic of Cyprus through the Research \& Innovation Foundation. Also, gaps observed for targeted earth observation geo-services related to the European and national policies are discussed. The paper ends with remarks dealing with the potential use of these geoservices for heritage management.

\section{Earth Observation Technological Shift}

Recent developments regarding big-data processing, have shifted the traditional applications of singlecomputer based earth observation to cloud-based. In this direction, the European Commission has funded the socalled Data and Information Access Services (DIAS) cloud-based platforms (Data and Information Access Services (DIAS, 2020), providing thus cloud access to the Copernicus data.

The DIAS platforms are developed when other private commercial entities have showcased the tremendous advantages of big-data cloud services for the end-users. The Google Earth Engine (GEE) is considered one widely known earth observation big-data cloud platform, whereas, different satellite data, can be processed online.

The Google Earth Engine@ (2015) is "a platform for petabyte-scale scientific analysis and visualisation of geospatial datasets". The engine is acting as a computing platform that runs on top of the Google's infrastructure, allowing end-users to access petabytes of earthobservation datasets, including the Copernicus data, around the globe (Fig. 1).

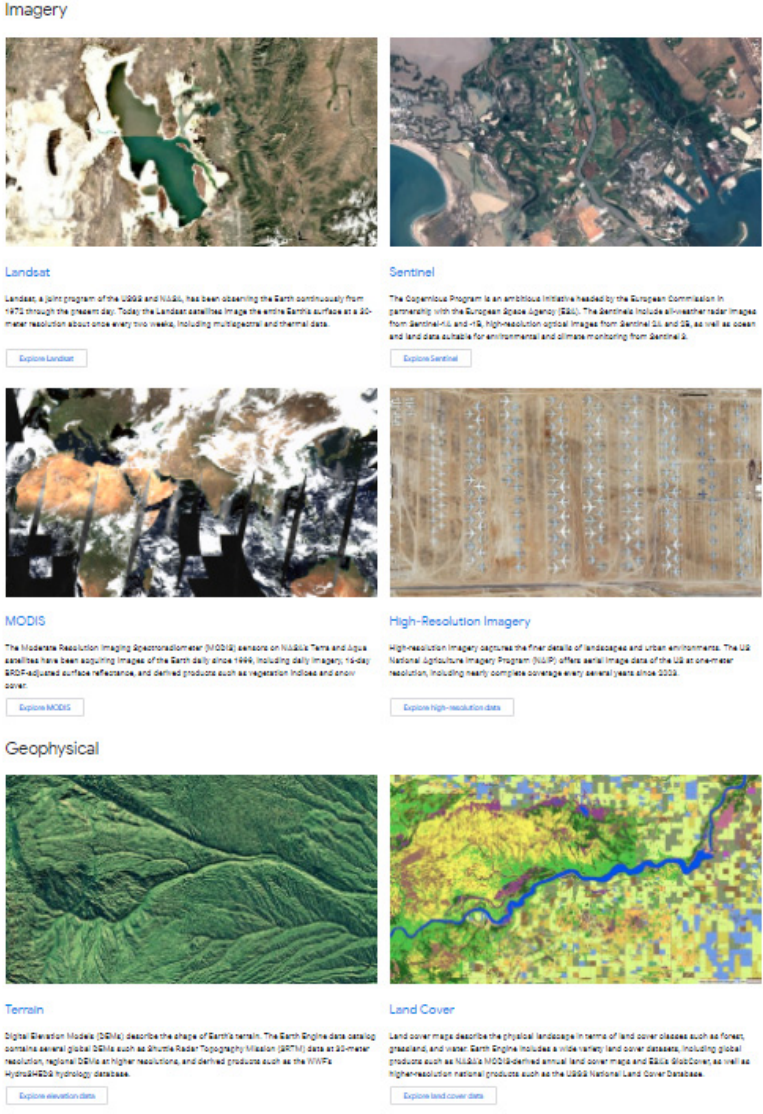

Figure 1: Screenshot from the Google Earth Engine data access (@Google).

The Google Earth Engine( $)$ includes various earth observation data and images before launching the first Sentinel sensors. The databased includes the Landsat series, while the end-users can upload other highresolution datasets for further processing.

The platform is expandable so that end-users can input (?) customised applications (e.g., image processing routines). The platform's access can be carried out in two ways: using the Core Editor or the Explorer. Explorer interface is a web access point to the platform that does not require any prior programming skills, while the Core Editor interface is more suitable for complex geospatial workflows (Fig. 2).

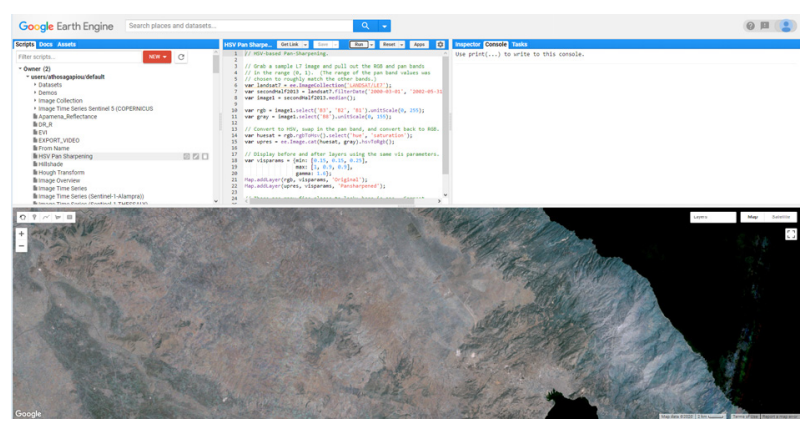

Figure 2: Screenshot from the Google Earth Engine Core Editor.

Similarly, other relevant cloud-based platforms are currently running to the Google Earth Engines, like the Amazon Web Services (2020). It should be mentioned 
that these cloud-based services explore machine and deep learning approaches, increasing thus the overall performance of image processing.

\section{Copernicus Services in Support of Cultural Heritage}

Very recently, in 2019, the European Commission has released the "Copernicus services in support to Cultural Heritage" report (Copernicus services in support to Cultural Heritage, 2020), that summarises the prospective implementation of the Copernicus Programme to cultural heritage applications. This report provides a European scale investigation, whereas several inputs from national agencies and other interested parties were reported. Even though cultural heritage is not directly linked to any of the existing Copernicus core services, the Copernicus related datasets can support some cultural heritage user requirements (see more in the next section).

The analysis of that report emphasised that only a small percentage $(7.5 \%)$ of the cultural heritage user requirements are already fully covered by Copernicus core services products. An additional $19.0 \%$ of user requirements have mentioned that are partially covered by existing Copernicus core services products without adaptation.

Adding to the Copernicus the Contributing Missions capabilities, these numbers increased by $50 \%$ of the user requirements (for fully covered), while an additional $14 \%$ could be partially covered. Therefore, half of the endusers requirements could be potentially supported by the downstream industry with access to very high-resolution data and/or very high revisiting time imagery, not available in the pool of Copernicus Contributing Missions. Overall, by using all Copernicus capabilities (core services products, Sentinels and Contributing Missions), $64 \%$ of cultural heritage end-user requirements could be covered.

Nonetheless, $36 \%$ of the end-user requirements, could not be covered by the Copernicus programme (Contributing Missions included). From this percentage, $7 \%$ cannot be covered due to the spatial or temporal limitations of the existing Copernicus Programme. In comparison, another $13 \%$ remains uncovered due to unfulfilled needs (i.e., sensors and/or wavelengths - such as hyperspectral, lidar - not available in the Copernicus Programme. Finally, $16 \%$ of the cultural heritage user requirements cannot be covered by satellite-based imagery at all. These needs require precise in-situ measurements and surveys (Ground Penetrating Radar (GPR), bathymetric surveys, etc.) or other complex valueadded products (e.g., assessment of sites frequentation pattern).

Concerning the monitoring of heritage sites from either natural or anthropogenic hazards, the report specifies that the existing Copernicus services focus mostly on cultural heritage rather than natural heritage sites. Hazard-related actions from the Copernicus Programme includes: (a) the hazard risk mapping of heritage sites that can be subjected to damage in cases of extreme meteorological events like floods or natural disasters such as earthquakes; (b) hazard early warning related to heritage sites that may be impacted/damaged by a geo-hazard; (c) hazard monitoring heritage sites for specific geo-hazards to support damage mitigation and damage assessment; and (d) hazard damage assessment of heritage sites due to a specific geo-hazard.

The capacity for performing these four categories of activities is critical for protecting and safeguarding heritage sites in Europe and worldwide. Commonly, when a geo-hazard occurs, civil protection agencies in charge of emergency response and mitigation focus first on human life protection and damage prevention on land use (e.g., critical infrastructure, farms, industries, etc.). In general, the safeguarding of cultural heritage is usually a secondary concern for civil protection agencies. However, some best practices have been introduced on a national level. In Italy for example, after major earthquakes that have hit the central part of the country in 2009 and 2016, a specialised body called "Funzione Beni Culturali" has been formed for securing the movable and immovable heritage. The two main representatives of this body are MiBACT (Italian Ministry of Cultural Heritage and Activities and Tourism) and Civil Protection. Such initiative declares a significant public commitment that can set the ground for a more extensive intervention framework on built Cultural Heritage in periods of hazards (Brazzetti \& Cuca, 2020).

\section{Cultural Heritage Applications: A Literature Review}

A literature review analysis, though the Scopus engine was performed in relation to the Copernicus programme and its contribution to cultural heritage. Scopus is the largest abstract and citation database of peer-reviewed literature: scientific journals, books, and conference proceedings.

The following search criteria were input in the Scopus database: "Copernicus AND archaeology"; "Copernicus AND cultural AND heritage"; "Sentinel AND archaeology" and "Sentinel AND cultural AND heritage". These keywords were selected to be found either in the title, the abstract, or the scientific journals' keywords within the Scopus database. This analysis's results are visualised in the figure below (Fig. 3), where a gradual increase in publications is observed, with an ever-increasing tendency from 2017 onwards. The use of Copernicus for cultural heritage was published in several scientific journals such as the "Remote Sensing MDPI", "Natural Hazards and Earth System Sciences", "Proceedings of SPIE" etc. Other findings, like documents by the author, documents by country or territory, and documents by funding sponsor are also shown in Figure 3.

Moreover, various risks that can affect cultural heritage, such as fires, earthquakes, landslides, soil erosion, floods, urbanisation, war conflicts or looting, have also been searched in the Scopus engine. Beyond the type of risk, the word "Satellite" was added, from 2010 to 2020. Figure 4 depicts the publications for the last decade (2010-2020). As shown, floods and earthquakes risks were by far the topic that attracted researchers' interest with 5162 and 5296 publications, followed by soil erosion, fires, and urbanisation (3587, 3206, and 2538 articles respectively). War conflicts and looting using satellite observation raised limited studies with only 39 articles in total. Also, a query for "Sentinel-1" and "Sentinel 2" has revealed a total number of more than 12000 articles indicating a significant wealth of research in exploiting the specific Copernicus sensors. 


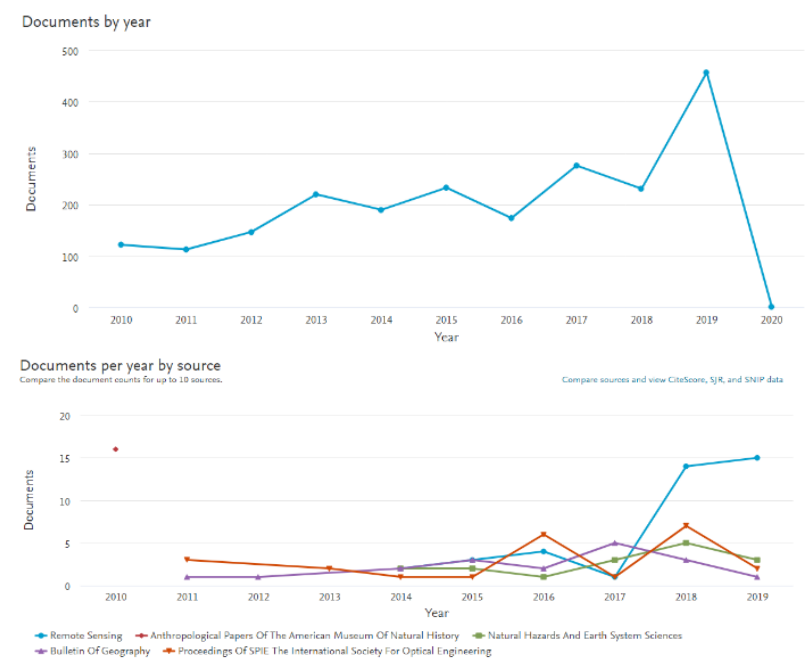

Figure 3: Scopus engine query results concerning the use of Copernicus and cultural heritage since 2010. Documents per year are shown on top while documents per year by source are visualised below-analysis for the NAVIGATOR project.

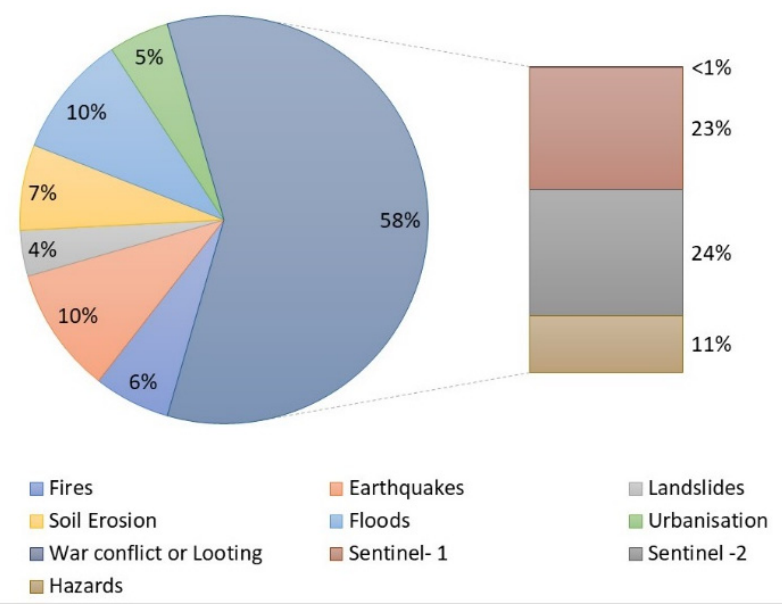

Figure 4: Total number of articles related to the study of various hazards with the support of satellite and space-based observations for the last decade (2010-2020) (results from Scopus engine database). Analysis of the NAVIGATOR project.

Specific queries at the Scopus engine such as "Looting and Earth Observation" or "Looting and Remote Sensing" and the "Earthquakes and Earth Observation" or "Earthquakes and Remote Sensing" have also been performed. The Scopus engine's literature review was then extracted and imported in the VOSviewer a software tool for creating maps based on network data, as shown in Figure 5 for looting and earthquakes risks, respectively. The different colours of this visual representation stand for a different thematic cluster, based on the keywords data of all articles found in the search engine. The size of each circle represents each cluster's size (i.e., how many times the specific term has been used). The links between each cluster are also visualised.

This desk-based analysis's overall results outlined the recent evolution of Earth Observation for applications related to cultural heritage.

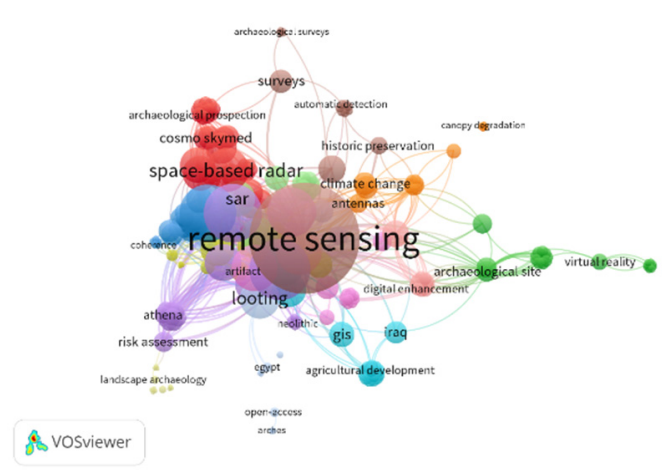

Figure 5: Visualisation of the scientific literature for looting. The database was extracted from the Scopus engine and visualised in the VOSviewer software. Analysis of the NAVIGATOR project.

\section{Earth Observation-based Applications}

In the past, remote sensing applications for cultural heritage have been populated in the scientific literature (Luo et al., 2019; Agapiou \& Lysandrou, 2015). The recent capabilities of the big-earth data cloud platforms have been already presented as well (Orengo et al., 2020). Several other studies have also used Copernicus radar and optical data for heritage management and risk assessment (Howland, Jones, Najjar, \& Levy, 2018; Agapiou et al., 2015; Cuca \& Agapiou, 2018).

In this section, we provide some of these examples, mainly focused on using the Copernicus Programme and other legacy data like the Landsat series. A recent example is Sentinel-1 images from monitoring land displacements after an earthquake event that occurred in the western part of Cyprus in 2015 (ref). The hightemporal revisit time of the Sentinel-1 images provides the opportunity to process images before and after an earthquake event. We can then blend this information with other geographical data using Geographical Information Systems to perform deformation analysis. Such damage proxy maps are critical for heritage management, especially after natural or man-made catastrophic events.

Figure 6 shows the coherence map as derived from the Sentinel-1 SAR images in ascending orbit and descending orbit using two pairs of Sentinel-1 images taken before and after the earthquake event. Figure 7 shows the unwrapped interferogram (Fig. 7a), the vertical displacement (Fig. 7b) and the coherence map (Fig. 7c) in the surroundings of a UNESCO World Heritage site in the western part of Cyprus (Agapiou \& Lysandrou, 2020).

Similarly, we can also explore the optical Sentinel-2 images for detecting urban areas in the vicinity of archaeological sites. Classification analysis of the multispectral optical data can be used to map the urban areas for extended regions. Using archive information like the Corine Land Use Land Cover program, the trend and the overall change in archaeological sites' vicinity can be estimated. In Figure 8 the red colour indicates the urban sprawl over the archaeological site of "Nea Paphos" and "Tombs of the Kings" in Paphos district, Cyprus (see yellow rectangles in Figure 8 left). The classification accuracy was estimated to be more than $86 \%$ using the random forest classifier. 

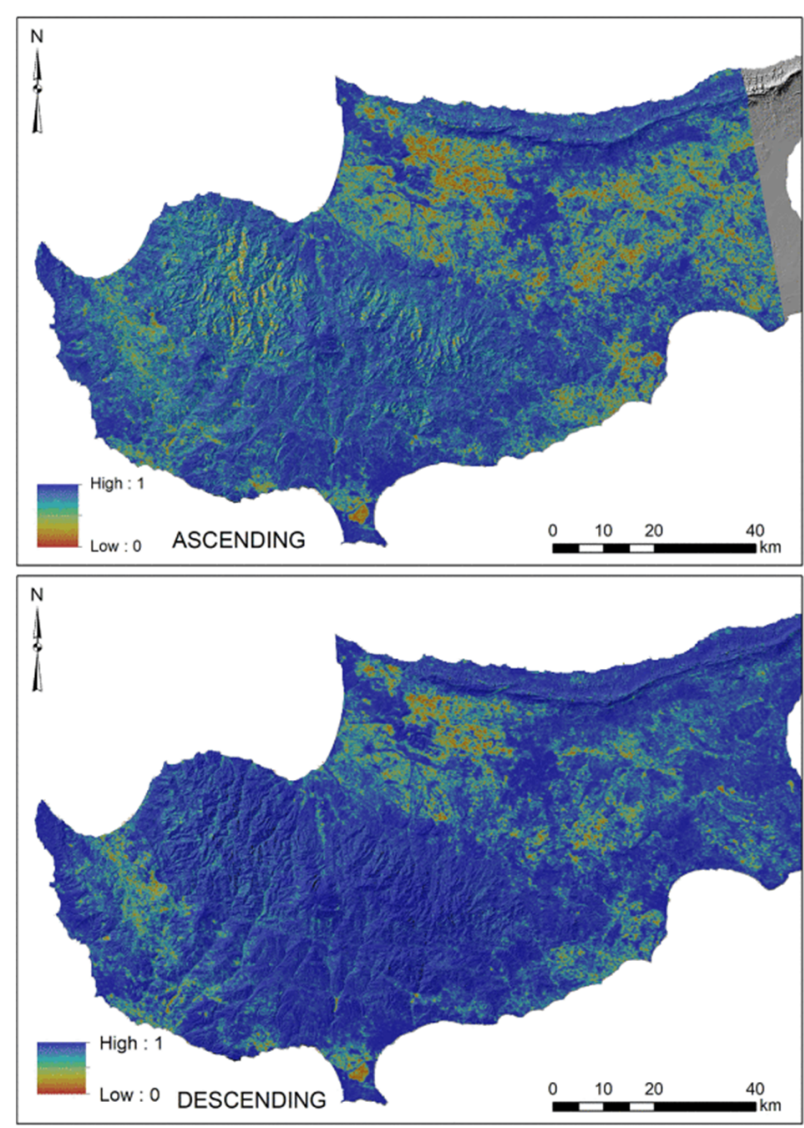

Figure 6: Coherence map as derived from the Sentinel-1 SAR images in ascending orbit and descending orbit. Analysis of the NAVIGATOR project (Agapiou \& Lysandrou, 2020).
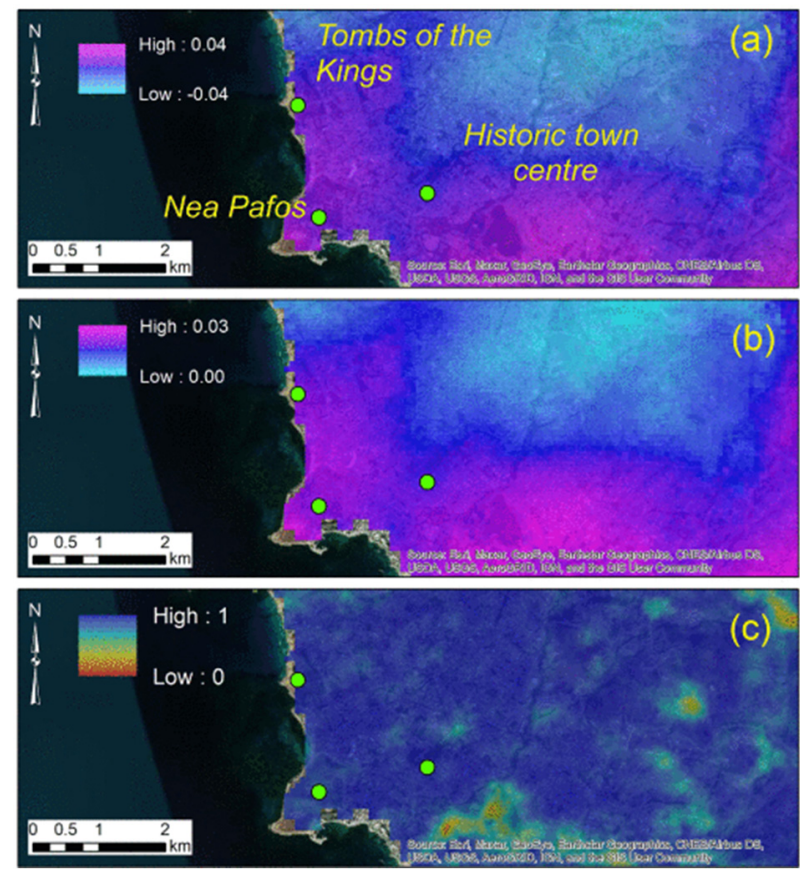

Figure 7: UNESCO World Heritage site in the western part of Cyprus (Agapiou \& Lysandrou, 2020): a) Unwrapped interferogram; b) Vertical displacements; c) Coherence map.

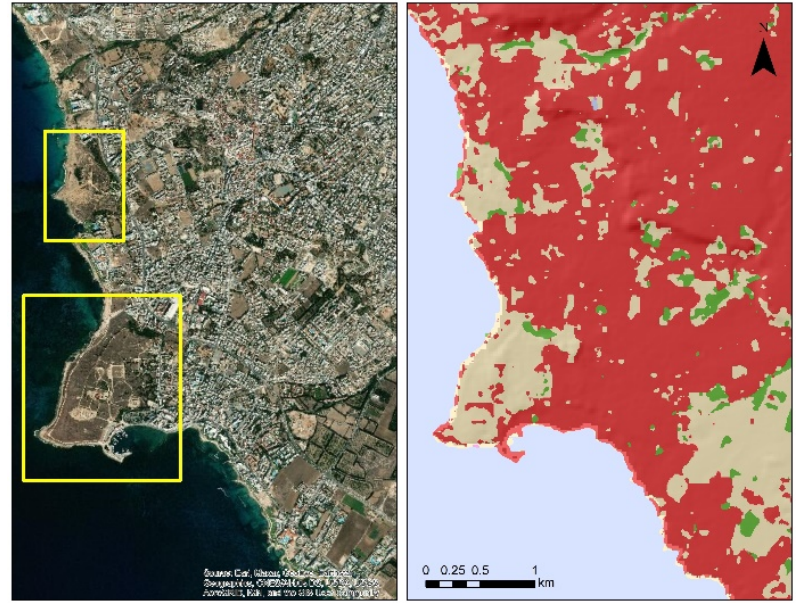

Figure 8: Classification results of a Sentinel-2 optical image over the Paphos town, western part of Cyprus (right). Urban areas are shown with red colour. On the left, two UNESCO World Heritage properties, namely the "Nea Paphos" and the "Tombs of the Kings" are shown with a yellow rectangle. Analysis for the NAVIGATOR project.

Other legacy satellite data, like those of the Landsat programme, can be explored to analyse historic buildings' thermal conditions. For this multi-temporal analysis, the macro-scale thermal anomalies can be further enhanced with higher spatial resolution and ground data, as shown in Figure 9. In the micro-scale level of observation ground sensors and observations on individual historic buildings can also be used as validation and calibration inputs of the satellite-based observations. The mean temperature over the municipality of Strovolos and Limassol, Cyprus, for a period covering from 2013-2020 are shown in Figures 10 and 11 , respectively.

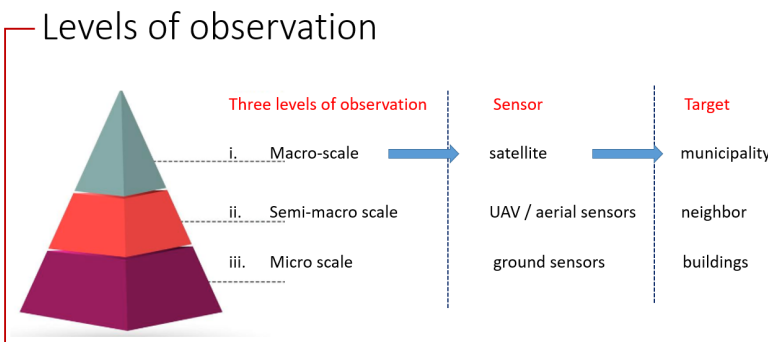

Figure 9: Observation levels for monitoring the thermal conditions of historic buildings. Analysis for the PERISCOPE project.

To better understand how earth observation and specific the Copernicus Programme can support cultural heritage, we should investigate cultural heritage needs. Figure 12 shows the disaster risk management (DRM) cycle, composed of six steps starting from the context, the identification of risk, the analysis, the evaluation and treatment and the monitoring phase (Agapiou, Lysandrou, \& Hadjimitsis, 2020). These steps are also known as preparedness and response phases and the recovery and mitigation actions. 


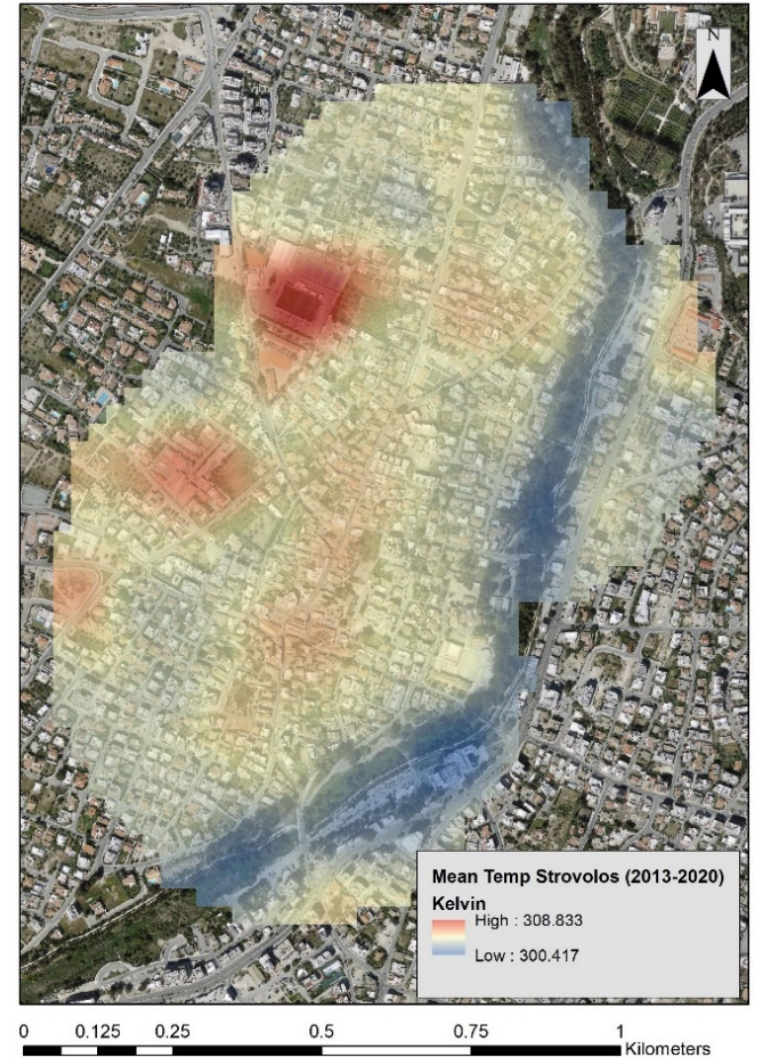

Figure 10: Mean temperature over Strovolos' municipality for a period from 2013-2020 using Landsat thermal images. Analysis of the PERISCOPE project.

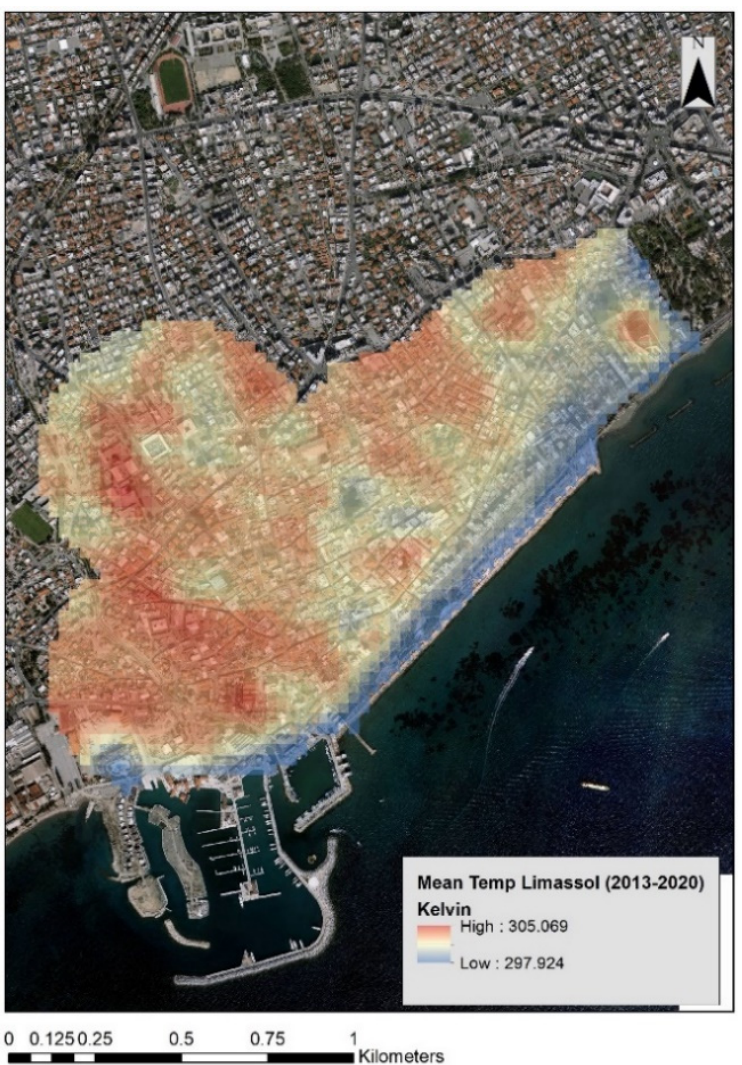

Figure 11: Mean temperature over the municipality of Limassol for a period from 2013-2020 using Landsat thermal images. Analysis of the PERISCOPE project.

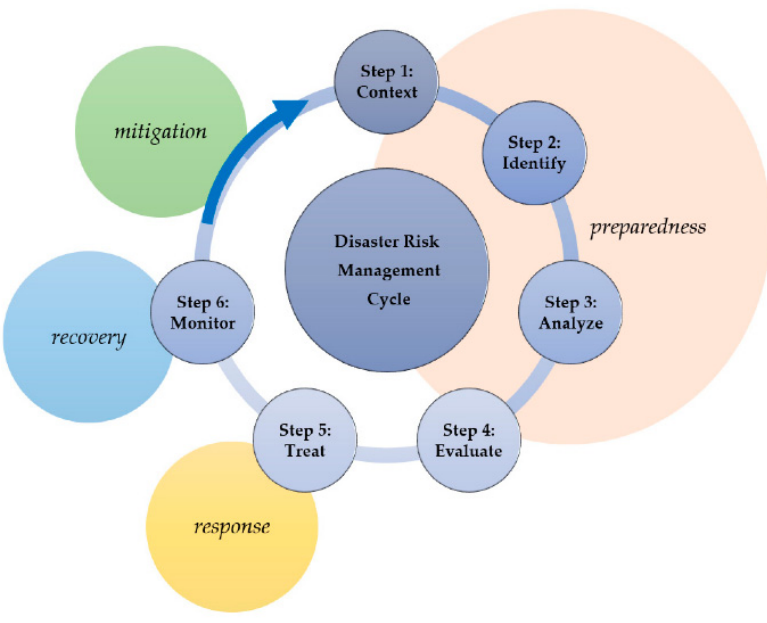

Figure 12: Disaster risk management (DRM) cycle for cultural heritage applications (Agapiou, Lysandrou, \& Hadjimitsis, 2020). Analysis of the NAVIGATOR project.

Earth observation can support the DRM cycle in various ways as this was also reported in (Agapiou, Lysandrou, \& Hadjimitsis, 2020). Figure 13 shows with green colour the potential contributions of the Copernicus Programme to DRM cycle in each of the six-steps:

Step 1. Understanding the context: Context information includes among others the physical environment of a site or monument. Such kind of information can be retrieved from the use of time-series satellite-based observations

Step 2. Identify risks: Agents of deterioration and loss, the various layers of enclosures, as well as the risk occurrence are defined. The use of earth observation images and image processing can be very important as satellite sensors can cover an extensive area and therefore oversee not only the archaeological site itself but also the surrounding area.

Step 3. Analyzing risks: this step quantifies the potential impact of all considered risks and any potential uncertainties of the analysis.

Step 4. Evaluation of risks: At this step, earth observation images can be used as inputs in the various models to evaluate the risks.

Step 5. Treating risks: Earth observation can be used as input layers of information for treating risks and provide systematic input to local stakeholders.

Step 6. Monitoring: Earth observation systematic monitoring may be used as a systematic tool of observation and monitoring purposes.

These steps are interconnected, and therefore any assumptions and uncertainties at any stage might influence the subsequent stages of the DRM cycle. The design and conceptualisation of a DRM cycle plan is a collective effort made by several parties that can provide specific information with local value, scientific knowledge for better characterisation of a site, and technological understanding. 


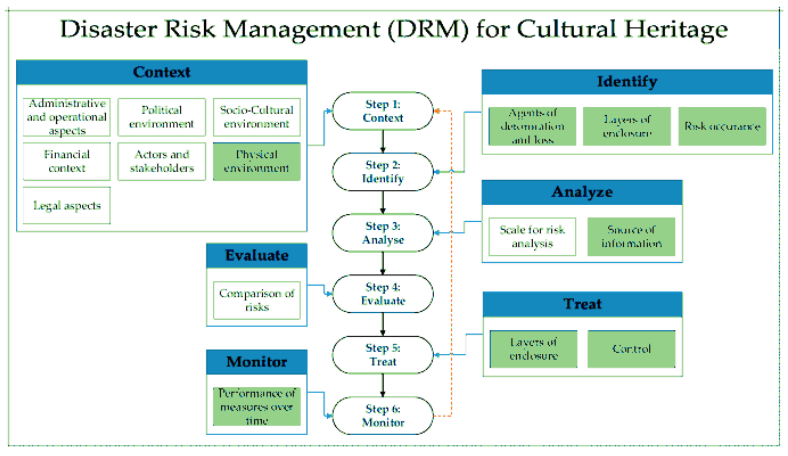

Figure 13: The potential contribution of satellite observation within the various steps of the risk management plan proposed by the International Centre for the Study of the Preservation and Restoration of Cultural Property (ICCROM) is highlighted in the green rectangle (Agapiou, Lysandrou, \& Hadjimitsis, 2020). Analysis of the NAVIGATOR project.

\section{Space-Based Applications for $\mathrm{CH}$ as a part of geospatial data policies}

Despite the increase of applications and case studies dealing with the Copernicus programme for cultural heritage, a gap is observed concerning adopting these methods as a systematic observation tool. National and regional authorities are pursuing a step forward through local INSPIRE Geo-portals, including Copernicus imagery and some specifically derived information supporting targeted actions. National and regional geo-portals are a product of coordinated European action to create a legal framework. This framework is used for the exchange, sharing, and access of interoperable (geo) spatial data and spatial data services, across different sectors and different levels of public authorities (European Parliament (EP), Directive 2007/2/EC, 2021). To include Sentinel imagery and derived products in such portals can be seen as an attempt to systematise geospatial information deriving from Earth Observation technologies. In this sense, the road for Cultural Heritage sites management could already be deemed as "paved" if the Theme "Protected sites" is considered. A specific INSPIRE theme dedicated to Cultural and Natural heritage could indeed serve not only for purposes of geo-location of specific monuments or areas under protection. It can also monitor their current state, vulnerability and potential threats that could be detected using integrated remote sensing technologies, including satellite imagery and products.

\section{Conclusions}

This paper presented some potentials of using earth observation (EO) sensors, including the Copernicus Programme data, with a focus on cultural heritage applications. The increased employment of space-based observations for monitoring purposes and risk assessment are some of these applications. The new technological shift observed in terms of image processing and cloud computing will impact the traditional way of EO implementations.

Earth observation can play an important role in the disaster risk management cycle (DRM) supporting local stakeholders and responsible agencies with tailoredmade products and different management phases. However, today, a gap still exists regarding the adoption of space-based sensors by national agencies to monitor cultural sites and monuments systematically.

One way of bridging this gap is to rely on organised and systematically updated infrastructures of open geospatial data where satellite-based data and services, like the Copernicus Sentinels, can provide timely and targeted products. These infrastructures can support cultural heritage preservation and management.

\section{Acknowledgements}

The paper is submitted under the NAVIGATOR project. The project is being co-funded by the Republic of Cyprus and the European Union's Structural Funds in Cyprus under the Research and Innovation Foundation grant agreement EXCELLENCE/0918/0052 (Copernicus Earth Observation Big Data for Cultural Heritage). Results related to the Landsat thermal analysis are part of the "Portal for heritage buildings integration into the contemporary built environment", in short PERISCOPE, co-financed by the European Regional Development Fund and the Republic of Cyprus through the Research \& Innovation Foundation. Grant Agreement INTEGRATED/0918/0034.

\section{References}

Agapiou, A., Lysandrou, V. \& Hadjimitsis, D. G. (2020). Earth Observation Contribution to Cultural Heritage Disaster Risk Management: Case Study of Eastern Mediterranean Open Air Archaeological Monuments and Sites. Remote Sens., 12, 1330. https://doi.org/10.3390/rs12081330

Agapiou, A., \& Lysandrou, V. (2020). Detecting Displacements Within Archaeological Sites in Cyprus After a 5.6 Magnitude Scale Earthquake Event Through the Hybrid Pluggable Processing Pipeline (HyP3) Cloud-Based System and Sentinel-1 Interferometric Synthetic Aperture Radar (InSAR) Analysis. IEEE Journal of Selected Topics in Applied Earth Observations and Remote Sensing, 13, 6115-6123. doi: 10.1109/JSTARS.2020.3028272

Agapiou, A., \& Lysandrou, V. (2015). Remote Sensing Archaeology: Tracking and mapping evolution in scientific literature from 1999-2015. J. Archaeol. Sci. Rep., 4, 192-200. https://doi.org/10.1016/j.jasrep.2015.09.010.

Agapiou, A., Alexakis, D. D., Lysandrou, V., Sarris, A., Cuca, B., Themistocleous, K., \& Hadjimitsis, D. G. (2015). Impact of Urban Sprawl to archaeological research: The case study of Paphos area in Cyprus. J. Cult. Herit., 16, 671-680. doi:10.1016/J.CULHER.2014.12.006

Amazon WebServices (2020). AWS | Cloud Computing - Servicios de informática en la nube. Retrieved January 27, 2021 from https://aws.amazon.com

Brazzetti, L., \& Cuca, B. (2020). Identification of Buildings Damaged by Natural Hazards Using Very High-Resolution Satellite Images: The Case of Earthquake in L'Aquila, Italy. In Hadjimitsis et al. (Eds), Remote Sensing for 
Archaeology and Cultural Landscapes (pp. pp 139-151). https://doi.org/10.1007/978-3-030-10979-0_9

Copernicus. (2020). Retrieved January 27, 2021 from https://www.copernicus.eu/en

Copernicus Services. (2020). Retrieved January 27, 2021 from https://www.copernicus.eu/en/copernicus-services

Copernicus services in support to Cultural Heritage (2020). Retrieved January 27, 2021 from https://www.copernicus.eu/sites/default/files/2019-06/Copernicus_services_in_support_to_Cultural_heritage.pdf

Cuca, B., \& Agapiou, A. (2018). Impact of land use change and soil erosion on cultural landscapes: The case of cultural paths and sites in Paphos district, Cyprus. Appl. Geomat., 10, 51. https://doi.org/10.1007/s12518-018-0237-z

Data and Information Access Services (DIAS) (2020). Retrieved January 27, 2021 from https://www.copernicus.eu/en/access-data/dias

European Parliament (EP), Directive 2007/2/EC: Establishing an Infrastructure for Spatial Information in the European Community (INSPIRE), last accessed Jan 2021 EUR-Lex - 32007L0002 - EN - EUR-Lex (europa.eu)

European Comission (2018). COM(2018) 447 final. Retrieved January 27, 2021 from https://eur-lex.europa.eu/legalcontent/EN/TXT/?uri=COM\%3A2018\%3A447\%3AFIN

Google Earth Engine Team. (2015). Google Earth Engine: A planetary-scale geospatial analysis platform. Retrieved January 27, 2021 from https://earthengine.google.com

Howland, M.D., Jones, I.W.N., Najjar, M., \& Levy, E.T. (2018).Quantifying the effects of erosion on archaeological sites with low-Altitude aerial photography, structure from motion, and GIS: A case study from southern Jordan. J. Archaeol. Sci., 90, 62-70. https://doi.org/10.1016/j.jas.2017.12.008

Luo, L., Wang, X., Guo, H., Lasaponara, R., Zong, X., Masini, N., Wang, G., Shi, P., Khatteli, H., Chen, F. (2019). Airborne and spaceborne remote sensing for archaeological and cultural heritage applications: A review of the century (19072017). Remote Sens. Environ., 232, 111280. https://doi.org/10.1016/j.rse.2019.111280

Orengo, A. H., Conesa, C. F., Garcia-Molsosa, A., Lobo, A., Green, S. A., Madella, M., \& Petrie, A. C. (2020). Automated detection of archaeological mounds using machine-learning classification of multisensor and multitemporal satellite data. Proceedings of the National Academy of Sciences Aug, 117(31) 18240-18250. https://doi.org/10.1073/pnas.2005583117

Satellites components. (2020). Satellites component | Copernicus. Retrieved January 27, 2021 from https://www.copernicus.eu/en/about-copernicus/infrastructure/satellites-component

Sentinel Overview. (2020). Missions - Sentinel Online. Retrieved January 27, 2021 from https://sentinel.esa.int/web/sentinel/missions

Sentinel-1. (2020). Missions - Sentinel Online. Retrieved January 27, 2021 from https://sentinel.esa.int/web/sentinel/missions/sentinel-1

Sentinel-2. (2020). Missions - Sentinel Online. Retrieved January 27, 2021 from https://sentinel.esa.int/web/sentinel/missions/sentinel-2 